\title{
Relevance of cancer cachexia models - muscle whole genome gene expression in human and animal cachexia
}

\section{Rogier L.C. Plas}

Wageningen Universiteit en Research

\section{Guido Hooiveld}

Wageningen Universiteit en Research

Renger F. Witkamp

Wageningen Universiteit en Research

Klaske van Norren ( $\square$ klaske.vannorren@wur.nl )

Wageningen Universiteit en Research https://orcid.org/0000-0002-6281-9455

\section{Research}

Keywords: Transcriptomics, micro-array, muscle, animal transcriptome, human transcriptome

Posted Date: October 26th, 2020

DOI: https://doi.org/10.21203/rs.3.rs-56696/v1

License: (a) (i) This work is licensed under a Creative Commons Attribution 4.0 International License. Read Full License 


\section{Relevance of cancer cachexia models - muscle whole genome gene}

\section{expression in human and animal cachexia}

\section{Rogier L.C. Plas ${ }^{1}$, Guido Hooiveld ${ }^{1}$, Renger F. Witkamp ${ }^{1}$, Klaske van Norren ${ }^{1}$}

Affiliations:

${ }^{1}$ Division of Human Nutrition and Health, Wageningen University, Wageningen, The Netherlands

\section{Corresponding author:}

\section{Dr. Klaske van Norren}

Klaske.vannorren@wur.nl

Wageningen University - AFSG - Human Nutrition and Health

P.O. Box 17, 6700 AA Wageningen

Stippeneng 4, 6708 WE Wageningen

Wageningen Campus I Building 124 (Helix)

+31317485093

\section{Abstract}

Background Cancer cachexia is a complex and multi-factorial syndrome. As currently available therapeutic options are limited, more in-depth knowledge on cachexia pathophysiology and the underlying molecular mechanisms remains warranted. Studies with animal models provide useful insights but they only mimic the human situation to a certain degree. Furthermore, there is heterogeneity in the design of published animal studies and outcomes. To further address this issue, we performed a comparative study analysing muscle whole genome gene expression of different cachexia studies in mice and human.

Methods We selected data sets from the NCBI Gene Expression Omnibus database containing muscle gene expression data measured by micro-array or RNA-sequencing, at least comprising a cachectic/tumour bearing group ( $n>3$ ) and a non-cachectic/control group ( $n>3$ ). This provided 12 datasets; 9 from mouse models and 3 human datasets. All datasets were quality checked, normalised and annotated. Datasets were merged and compared at different levels. General similarity and differences in gene expression were determined using ordered list analysis and principal component analysis (PCA). Moreover, similarities and differences at pathway level were studied by applying gene set enrichment analysis (GSEA) of KEGG pathways.

Results Animal models displayed similarities to each other and to human datasets at different levels and with different processes. At the gene level, a similarity analysis indicated little similarity between the animal models and the human datasets, while animal models showed high similarity. Only one of the C26 mice models (GSE121972) showed significant similarity to more than one human dataset. Moreover, one human dataset comparing cachectic and non-cachectic cancer patients showed no similarity to any of the other datasets. PCA results indicated that a xenograft model showed most different expression from the other datasets and the Lewis lung carcinoma model to be least different from the human datasets. GSEA results showed four pathways clearly standing out across experiments with downregulation of oxidative phosphorylation and thermogenesis pathway, and upregulation of the proteasome and RNA transport pathway. However, these pathways were not consistently changed in the human datasets.

Conclusions Our comparative analysis showed that there is currently no basis to define a preferred animal model for human cachexia. More human datasets containing proper controls are needed. Repetition of the current analysis upon publication of additional human datasets is warranted.

\section{Keywords}

Transcriptomics, micro-array, muscle, animal transcriptome, human transcriptome 


\section{Introduction}

Cachexia is a multifactorial syndrome characterised by disease-induced progressive loss of muscle and/or fat mass. It is often present in patients with chronic kidney disease, heart failure, COPD or cancer. In many patients cachexia contributes to reduced treatment efficacy and quality of life. Experts generally agree that cachexia should be treated with a multimodal approach addressing dietary intake, systemic inflammation and physical activity [1,2]. These different treatment components are often studied using different mouse models. The current study aimed to evaluate the relevance of different animal models mimicking human cancer cachexia by comparing muscle gene expression in different mouse models with that in human cancer cachexia. To this end, we used a big data approach by analysing publicly available datasets present in the GEO database containing whole genome gene expression measured by either microarray or sequencing.

Different types of tumours can cause cachexia both directly, by secreting cachexia-inducing factors, and indirectly, by triggering a systemic inflammatory process. To simulate human cancer cachexia and investigate treatment options, different types of animal models exist [3-10]. The traditional and most common models, i.e. C26 or Lewis Lung Carcinoma (LLC), use direct injection of in vitro cultured tumour cells $[3,4,6-8,10]$. These are usually injected in the flank or muscle of the mouse where they grow into a solid tumour. More recent are models with genetic modifications causing spontaneous tumours [5], models using adenoviruses to induce tumour development [9], and xenograft models where tumour cells are harvested from human tumours and implanted in mice [8]. The difference between the models not only lies in the type of tumour induction. Different models have different time-spans ranging from 14 days to 8.5 weeks after tumour induction. In addition to the different types of models, experimental conditions may also vary between experiments using the same model. Despite these differences, all these models aim to mimic human cancer cachexia. To assess animal model relevance, meta-analyses and systematic reviews have been performed on differences and commonalities of molecular processes between mice and men [11,12]. However, no studies directly compared data of molecular processes driving the cachexia in animals or humans, which makes the relevance of the animal models used a subject for debate. Therefore, our aim was to elucidate possible differences and commonalities in molecular processes in muscle tissues from different cachexia samples.

Whole genome gene expression of muscle tissue has been measured in several cachexia studies using micro array analysis or RNA sequencing. Upon publication of these whole genome gene expression studies, raw data is often made available via the NCBI Gene Expression Omnibus database. By comparing the different datasets using a big data approach, differences and similarities between models and experimental conditions can be examined. Moreover, possible knowledge can be gathered on underlying processes causing muscle wasting and on possible differences between animal models for cancer cachexia and human cancer cachexia.

\section{Materials and Methods}

\section{Tumour model Dataset inclusion}

We selected data series present in the NCBI Gene Expression Omnibus database using the keywords cachexia or (cancer AND skeletal muscle). From these, we selected those series containing muscle gene expression data of a cachectic/tumour bearing group $(n>3)$ and a non-cachectic/control group ( $n>3)$. This provided 12 data series; 9 from mouse models and 3 human datasets (figure 1 and table 1 ).

\section{Data processing}

All datasets were quality checked, normalised and annotated with the latest annotations available using $\mathrm{R}$ statistical computing software (https://www.r-project.org/). The flowchart in figure 1 summarizes data selection and processing work flow. Three different measurement modalities were distinguished; (1) Affymetrix arrays, (2) Illumina bead-chip arrays and (3) Illumina HiSeq RNA-sequencing results. (1) Data obtained with Affymetrix arrays were quality checked and normalized using the robust multi-array analysis (RMA) algorithm [13] as implemented in the Bioconductor package AffyPLM. Probe sets were identified with genome information according to Dai et al. [14] based on annotations provided 
by the Entrez Gene database (custom CDF v23). (2) Data collected with Illumina beadchip arrays were background corrected and normalized using quantile normalization (neqc) [15]. Subsequently, unspecific probes were removed [16] (3) Illumina HiSeq RNA-sequencing results were filtered for genes having an expression level greater than 10 counts. Next to that, the library size and the experimental design was taken into account and corrected for, using the Bioconductor package edgeR $[17,18]$. Subsequently, library size differences were adjusted using the trimmed mean of M-values normalization method [19], implemented in the Bioconductor package edgeR [18]. Counts were then logtransformed and the observed mean-variance trend was converted into precision weights by the voom function[20] in the Bioconductor package limma [21].

After normalization, all different data types were analysed similarly. Differential expression of probe sets (genes) was determined using linear models (package limma) and an intensity-based moderated t-statistic [21,22]. For each data set, samples from tumour bearing animals/patients were compared to a healthy control group, with one exception: in GSE85017 a comparison between cachectic and non-cachectic cancer patients was made, because this dataset did not contain a non-cancer control group. Genes with a $p$-value of $p<0.01$ were considered to be significantly differing between groups. Individual sample values were scaled based on full groups to enable comparison of samples obtained in separate experiments. Mouse and human expression data was merged based on the gene homology database obtained from the Mouse Genome Informatics website, The Jackson Laboratory, Bar Harbor, Maine, accessible via the World Wide Web (URL: http://www.informatics.jax.org) [download September, 2017]. For all subsequent analyses, only genes measured in all experiments were taken into account, which resulted in a final dataset of 7694 genes and 168 samples.

Data analysis

All data analysis was performed in R. Data visualisation was done using ggplot2 and corrplot [23,24]. For similarity analysis we used the Bioconductor R library OrderedList [25]. Here, we used ranked listed based on the t-test statistic to compare all experiments in a pairwise fashion. Similarity scores with $p<0.05$ were normalized and visualized in an association matrix using corrplot and in a network plot using Cytoscape [26]. To assess differences between experiments, a sparse principal component analysis (sPCA) was performed available in the mixOmics package [27,28]. Using this sPCA, we reduced the dimensions and identified the top genes responsible for variation between the different datasets. Based on the number of different experimental types we selected the number of components to be $(n-1)$ and we specified to keep the 100 genes most responsible for the variation within each component. Changes in individual genes were related to changes in pathways by gene set enrichment analysis (GSEA)[29] using the subset of metabolic and signalling pathways retrieved from the expert-curated Kyoto Encyclopedia of Genes and Genomes (KEGG) database [30]. For each comparison, genes were ranked on their t-value that was calculated by the empirical Bayes method. Statistical significance of GSEA results was determined using 10,000 permutations. GSEA and visualization was performed using the Bioconductor package clusterProfiler [31]. To assess variation between separate samples of all datasets, an sPCA with 6 components of each 25 genes was performed on gene expression, normalized per experiment, of individual samples of all experiments $(n=168)$.

\section{Results}

\section{Included datasets}

We identified data sets from five different animal models that met our criteria for inclusion: two inoculation models; C26 $(n=5)$ and Lewis Lung Carcinoma (LLC), one adenovirus- induced model (non-small cell lung cancer [NSCLC]), one spontaneous model (pancreatic cancer) and one xenograft model. General model characteristics differ in several aspects. The C26 model and the human xenograft model are rather acute models with a duration between $14-25$ days between inoculation and sacrifice. The LLC model has a slightly longer experimental duration of 4 weeks, and the NSCLC and pancreatic cancer have a duration of $>8$ weeks. Moreover, different muscle types were used for analysis in the animal models. 
Next to this, we included 3 human datasets, one of which containing whole genome gene expression data of $m$. rectus abdominis samples of cachectic or non-cachectic pancreatic and colorectal cancer patients, one containing quadriceps samples of upper gastrointestinal cancer (UGI) patients and healthy controls, and one containing $\mathrm{m}$. rectus abdominis samples of UGI patients and weight stable (WS) patients undergoing surgery for benign, non-inflammatory conditions.

\section{Number of significantly changed gene transcription activities}

Analysis of the number of significantly changed gene transcription activities in each experiment showed that there were large differences in the magnitude of tumour effects on the muscle (figure 2). The C26 model was found to be overall the most invasive model affecting expression of up to 56\% (GSE56555) of all genes. However, this model also showed considerable between-study variation, as in the apparently least invasive study (GSE63032) transcription of only $16 \%$ of all genes was changed. The other mouse models showed on average a lower number of changed gene transcription activities. The adenovirus-induced non-small cell lung cancer (NSCLC, GSE107470) affected 31\%, the Lewis Lung Carcinoma (LLC, GSE114820) affected 29\%, the human xenograft model (GSE80081) affected 16\%, and the spontaneous pancreatic cancer (GSE81931) affected transcription of $12 \%$ of all genes. Compared to these mouse models, human datasets showed only very mild effects with $6 \%, 2 \%$ and $1 \%$ of all genes being affected in transcription (respectively GSE34111, GSE85017 and GSE18832).

Similarity analysis

Subsequently, we created a similarity network and matrix (figure 3 and 4) based on our pair-wise similarity analysis. Results showed that the highest similarity is among the C26 models and that only very minor similarities are found between the mouse models and the human models. Surprisingly, the similarity of two skeletal muscle samples from the same experiment (the $m$. soleus and $m$. extensor digitorum longus of [GSE121972]) was lower than the similarity between the transcriptome of four other experiments including the xenograft and spontaneous pancreatic cancer model. Moreover, there was little to no similarity between the human experiments. The human dataset comparing noncachectic with cachectic cancer patients showed no significant similarity to other datasets at all.

\section{Sparse Principal Component Analysis Expression Differences}

To assess differences between datasets, we performed a sparse Principal Component Analysis with 6 components of 100 genes, together explaining a total variance of $86 \%$. The first and second principal component clearly explained the largest separation with the human and C26 models clearly clustering together (figure 5 A). Component 3, 4 and 5 separated respectively the NSCLC, LLC and PAN models from the other models and component 6 explains variance within the C26 models (figure $5 \mathrm{~A}$ ). However, the explained variance in component 3:6 is less than half that of component 1 and 2 (figure $5 \mathrm{~B}$ and $\mathrm{C}$ ). In a heat map depicting expression of all genes present in the 6 components of the SPCA, the hierarchical clustering shows that the xenograft model is clearly separated from the other experiments and that the model closest to the human experiments is the LLC model (figure $5 \mathrm{D}$ ).

\section{Gene Set Enrichment Analysis}

When looking at the KEGG pathway enrichment, four pathways seemed to be important in all experiments; the proteasome and RNA transport pathway were both strongly upregulated in most experiments, while the oxidative phosphorylation and thermogenesis pathway were strongly downregulated in most experiments (figure 6). Interestingly, the effects on these pathways were quite uniform along all animal models, but differed to some extent between the human experiments with the Proteasome and RNA transport pathway being downregulated in the human $m$. quadriceps (GSE34111). When looking at the change in expression of the genes in these separate pathways (figure 7), it became clear that different models had differential effects on these four important pathways. In the most upregulated proteasome pathway and the downregulated oxidative phosphorylation and thermogenesis pathway, the human experiments clustered together, while separating from the mouse models. Regarding the RNA transport pathway, some overlap was found between the human experiments and the LLC, and NSCLC animal models. The xenograft model showed a distinct response in all but the proteasome pathway since it splits off first in the hierarchical clustering. When looking at correlation of normalized expression values for all experiments (figure 8 ), the human $m$. 
quadriceps shows a clearly distinct response correlating negatively with the other human datasets and only correlating positively with the LLC model. Moreover, clear correlations between the C26 models were visible.

\section{SPCA of separate samples}

We assessed differences between samples and inter-experimental variation using a sPCA with 6 components of each 25 genes explaining a total variance of $48 \%$. In the hierarchical clustering of the heatmap created with genes present in each of the components, some clear clusters become visible indicating distinct expression in some experiments (supplemental figure 2). The NSCLC (GSE114820) and LLC (GSE107470) model cluster together [left side of the heatmap] and there is a cluster of the xenograft (GSE80081) model together with one of the C26 models (GSE24112) [just right of the center of the heatmap]. However, the analytical platform may also be a dominating determinant as the data sets from the NSCLC and LLC models both contained Illumina-based RNA-seq data, while the xenograft and C26 quadriceps were generated with Illumina bead chips. Within the group of data sets generated with Affymetrix arrays, a clear separation between tumour-bearing animals [right of the xenograft/C26 cluster] and control animals [left of the xenograft/C26 cluster] of the other C26 models and the pancreatic cancer model was observed. Moreover, we see that most human controls are clustered together with the control animals. At the same time, no specific clustering of data from cancer patients with those from tumour bearing animals was observed. For the dataset with cachectic/non cachectic cases also no clear separation between cachectic- and non-cachectic cases was seen. Overall, human samples showed considerably more variation compared to those from the animal models and no clear separation became visible between datasets from control and cancer patients used in our analysis.

\section{Discussion}

Different models are in use to evaluate possible therapeutic strategies in cachexia, like those based on nutritional, exercise and pharmacological intervention. The present study aimed to determine the relevance of mouse models used to mimic human cachexia, by comparing their effects on muscle gene expression, both between models and with data from human cancer cachexia studies. Publicly available datasets like those present in the GEO database are increasingly offering opportunities for such meta-analyses without the need to perform additional (animal) studies. Overall, our analysis revealed some similarities between animal models and, to lesser extent, to human datasets, in particular with respect to biological process level.

On gene level, analysis indicated only little similarity between the data from animal models and those from human datasets, while more similarities were found between the animal models. Only the $m$. extensor digitorum longus and $m$. soleus data from one of the C26 models (GSE121972) showed significant similarity to more than one human dataset. Moreover, the human dataset without healthy controls showed no similarity to any of the other datasets. However, principal component analysis to examine the specific differences between datasets revealed that the xenograft model showed the most different expression pattern compared to those of the other datasets. Here, the LLC model seems the least different from the human datasets based on clustering in the sPCA heatmap (figure 5.5 D). These results give a good impression of general similarities and differences between data obtained from animal models and those from human cachectic patients. However, for analysis of relevant molecular processes, GSEA results are preferred. With GSEA, four pathways were found to be clearly standing out. A clear downregulation of oxidative phosphorylation was observed in all animal models, which is in line with literature data [32]. However, this downregulation was only seen in the human $m$. quadriceps, while in the other human datasets, oxidative phosphorylation was not significantly enriched. For the thermogenesis pathway, a clear downregulation was seen in all animal models, whereas also here only the human $m$. quadriceps data showed significant downregulation. The downregulation in muscle thermogenesis is surprising since literature suggests an important role for increased thermogenesis during cachexia, not only in brown adipose tissue [33] but also in muscle [34]. When considering upregulated pathways, the proteasome pathway appeared to be strongly upregulated which is in line with literature [35]. However, the term proteasome is most frequently used in combination with the ubiquitin system [12,36,37]. In our analysis, we do not see the ubiquitin mediate proteolysis to be significantly enriched in any of the datasets. This is in line with previous suggestions that the ubiquitin-proteasome system is not as important in cancer-induced cachexia as previously thought [38]. Another 
relevant pathway is the strongly upregulated RNA transport pathway which is the only pathway where human datasets cluster together with some animal models (NSCLC and the LLC model). In this pathway, Eukaryotic translation initiation factor 4E binding protein 1 [EIF4EBP1] seems to be an important factor being highly downregulated (figure 7 B). Interestingly, this gene is mostly referred to in the context of its influence on the mTOR and AKT pathways in cachexia, whereas we obtained no indication of importance of either the mTOR nor the AKT pathway in our analysis (supplemental figure 1) [39]. Finally, some pathways came out of the human datasets without apparently being relevant in the animal models. For example, the RNA degradation was upregulated in both human $m$. rectus abdominis samples, while not significantly found to be enriched in any of the animal models (supplemental figure 1).

In line with literature, we see that the measurement platform markedly influenced clustering based on individual gene expression of separate samples [40]. This occurred despite the fact that we used the mixOmics analysis package correcting for differences in platform and experiment $[27,28]$. However, we do not see platforms clustering together in analyses on differential expression on gene or pathway level making these comparisons still highly relevant. Another limitation of this analysis is that the merge of all data from different platforms causes some data loss.

This is the first study on muscle gene expression in cachexia directly comparing whole genome gene expression data from different experiments. This type of analysis is getting more important due to the large increase in raw data made available and is already used for analysis of tumour gene expression in different types of cancer [41-44]. However, in case of muscle gene expression in cancer, the number of available data sets are still limited, making a repetition of this analysis upon publication of new data highly relevant. Unfortunately, phenotypic (meta-) data was often not present or insufficient with the datasets, making integration with other data like body weight loss and gene expression not possible for this analysis. Moreover, integration of different omics data like protein expression might be highly relevant to also gain insight into post-transcriptional processes important in cancer cachexia [45].

In conclusion, this study allows to do some suggestions, based on the currently available data, on the relevance of different models mimicking human cancer cachexia. Most importantly, we do not see one model out-performing other models. Each model shows its own differences and similarities to the publicly available human datasets. Thus, the choice for a specific model should be based on several characteristics. Most important is the duration of the experiment which is specifically important when looking at type of intervention; mild interventions might need experiments with longer duration while pharmacologic experiments could do with shorter duration. Moreover, the main targeted outcomes should also be considered since different experiments show similarity to the human situation with respect to different levels and pathways. Taken together, more human datasets containing proper controls are needed before we can draw firm conclusions on mechanisms involved and the usefulness of different models based on a big-data analysis of muscle whole genome gene expression.

\section{Abbreviation list}

$\begin{array}{ll}\text { AKT } & \text { acronym for protein kinase B, also known as PKB } \\ \text { C26 } & \text { Colon-26 Carcinoma } \\ \text { GEO } & \text { Gene Expression Omnibus } \\ \text { GSEA } & \text { gene set enrichment analysis } \\ \text { KEGG } & \text { Kyoto Encyclopedia of Genes and Genomes } \\ \text { LLC } & \text { Lewis Lung Carcinoma } \\ \text { mTOR } & \text { mammalian target of rapamycin } \\ \text { neqC } & \text { 'R' script name for quantile normalization } \\ \text { NSCLC } & \text { non-small cell lung cancer } \\ \text { PCA } & \text { principal component analysis } \\ \text { RMA } & \text { multi-array analysis } \\ \text { SPCA } & \text { sparse principal component analysis } \\ \text { UGI } & \text { upper gastrointestinal cancer } \\ \text { WS } & \text { weight stable }\end{array}$




\section{Declarations}

Ethics approval and consent to participate: not applicable.

Consent for publication : not applicable

Availability of data and material: R scripts have been added to make the analysis used available

Author contributions: RP, GH, RW and KvN did the concept and design of the study. Acquisition, analysis and interpretation of the data were carried out by RP, GH and KvN. Drafting of the manuscript was performed by RP, GH, RW and KvN. All authors revised the manuscript critically for important intellectual content and approved the final manuscript.

Conflict of interest: The authors declare no competing interests.

Funding: research was funded by the Wageningen University

Acknowlegdements: The authors would like to thank all the authors that contributed to the data as available in the GEO database and used for this study 
Tables and Figure captions

Table 1 - Overview of basic characteristics of included datasets. *Control group in GSE85017 consisted of non-cachectic cancer patients

\begin{tabular}{|c|c|c|c|c|c|c|c|}
\hline $\begin{array}{l}\text { GEOCODE } \\
\text { ABREVIATION }\end{array}$ & STUDY & MODEL & CONTROL & TUMOUR & DURATION & MUSCLE & PLATFORM \\
\hline $\begin{array}{l}\text { GSE24112 } \\
\text { C26 Quad }\end{array}$ & $\begin{array}{l}\text { Bonetto A et al., } \\
\text { PloS ONE, } 2015\end{array}$ & Mouse - C26 & 4 & 4 & $24 d$ & Quadriceps & Illumina MouseWG-6 v2.0 \\
\hline $\begin{array}{l}\text { GSE } 48363 \\
\text { C26 Gas+Plan }\end{array}$ & $\begin{array}{l}\text { Cornwell EW et al., } \\
\text { PloS ONE, } 2014\end{array}$ & Mouse - C26 & 3 & 3 & $25 d$ & $\begin{array}{l}\text { Gastrocnemius and } \\
\text { Plantaris mix }\end{array}$ & Affymetrix Mouse Gene 1.0 ST \\
\hline $\begin{array}{l}\text { GSE56555 } \\
\text { C26 Tib }\end{array}$ & $\begin{array}{l}\text { Judge SM et al., } \\
\text { BMC Cancer, } 2014\end{array}$ & Mouse - C26 & 4 & 3 & $\sim 26 \mathrm{~d}$ & Tibialis Anterior & Affymetrix Mouse Gene 1.0 ST \\
\hline $\begin{array}{l}\text { GSE63032 } \\
\text { C26 Gastr }\end{array}$ & $\begin{array}{l}\text { Shum AM et al., } \\
\text { Physiol. Genomics, } 2015\end{array}$ & Mouse - C26 & 3 & 3 & $14-19 d$ & Gastrocnemius & Affymetrix Mouse Gene 1.0 ST \\
\hline $\begin{array}{l}\text { GSE121972 } \\
\text { C26 EDL / SOL }\end{array}$ & $\begin{array}{l}\text { Plas RLC, } \\
\text { journal } 2018\end{array}$ & Mouse - C26 & 6 & 6 & $19 d$ & $\begin{array}{l}\text { Soleus \& Extensor } \\
\text { Digitorum Longus }\end{array}$ & Affymetrix Mouse Gene $1.1 \mathrm{ST}$ \\
\hline $\begin{array}{l}\text { GSE114820 } \\
\text { LLC Gastr }\end{array}$ & $\begin{array}{l}\text { Blackwell T et al., Physiol } \\
\text { Genomics, } 2018\end{array}$ & Mouse - LLC & 8 & 8 & 4 wk & Gastrocnemius & $\begin{array}{l}\text { Illumina HiSeq } 2500 \text { (Mus } \\
\text { musculus) }\end{array}$ \\
\hline $\begin{array}{l}\text { GSE107470 } \\
\text { NSCLC Gastr }\end{array}$ & $\begin{array}{l}\text { Goncalves M et al., PNAS, } \\
2018\end{array}$ & $\begin{array}{l}\text { Mouse - non small cell lung cancer } \\
\text { (adenovirus induced) }\end{array}$ & 5 & 5 & $8.5 \mathrm{wk}$ & Gastrocnemius & $\begin{array}{l}\text { Illumina HiSeq } 4000 \text { (Mus } \\
\text { musculus) }\end{array}$ \\
\hline $\begin{array}{l}\text { GSE51931 } \\
\text { PAN BicFem }\end{array}$ & $\begin{array}{l}\text { Gilabert M et al., J Cell } \\
\text { Physiol, } 2014\end{array}$ & $\begin{array}{l}\text { Mouse - Pancreatic cancer } \\
\text { (spontaneous) }\end{array}$ & 3 & 3 & $8-12 w k$ & Biceps Femoris & Affymetrix Mouse Gene 1.0 ST \\
\hline $\begin{array}{l}\text { GSE80081 } \\
\text { XEN Quad }\end{array}$ & $\begin{array}{l}\text { Fukawa T et al., Nat Med, } \\
2016\end{array}$ & $\begin{array}{l}\text { Mouse - Human Xenograft } \\
\text { (RXF393 vs SKRC39) }\end{array}$ & 3 & 3 & $22 \mathrm{~d}$ & Quadriceps & Illumina MouseWG-6 v2.0 \\
\hline $\begin{array}{l}\text { GSE85017 } \\
\text { HUM_NC RectAbd }\end{array}$ & $\begin{array}{l}\text { Narasimhan A et al., JCSM, } \\
2017\end{array}$ & $\begin{array}{l}\text { Human - Pancreatic and Colorectal } \\
\text { cancer (cachectic vs non cachectic) }\end{array}$ & $\begin{array}{c}19 \\
*\end{array}$ & 21 & $40-83$ y & Rectus Abdominis & $\begin{array}{l}\text { Affymetrix Human } \\
\text { Transcriptome Array } 2.0\end{array}$ \\
\hline $\begin{array}{l}\text { GSE34111 } \\
\text { HUM Quad }\end{array}$ & $\begin{array}{l}\text { Gallagher I et al., } \\
\text { Clin Cancer Res, } 2012\end{array}$ & $\begin{array}{l}\text { Human - upper gastrointestinal } \\
\text { cancer (cancer vs control) }\end{array}$ & 6 & 12 & $\sim 65 \mathrm{y}$ & Quadriceps & $\begin{array}{l}\text { Affymetrix GeneChip Human } \\
\text { Genome U133 Plus } 2.0\end{array}$ \\
\hline $\begin{array}{l}\text { GSE18832 } \\
\text { HUM RectAbd }\end{array}$ & $\begin{array}{l}\text { Stephens NA et al., } \\
\text { Genome Med., } 2010\end{array}$ & $\begin{array}{l}\text { Human - upper gastrointestinal } \\
\text { cancer (cancer vs control) }\end{array}$ & 3 & 18 & $\begin{array}{l}51 \text { y (control) } \\
65.5 \text { y (cancer) }\end{array}$ & Rectus Abdominis & $\begin{array}{l}\text { Affymetrix GeneChip Human } \\
\text { Genome U133 Plus } 2.0\end{array}$ \\
\hline
\end{tabular}


Figure 1 - Flowchart of data series inclusion and data processing.

Figure 2 - Number of significantly changed genes $(p<0.01)$ per experiment based on the complete dataset containing gene expression of 7694 genes present in all datasets.

Figure 3 - Network plot of similarity scores. Line width represent strength of similarity. Only similarities with $p<0.05$ are shown.

Figure 4 - Similarity matrix showing normalized similarity with $p<0.05$. All similarity scores were normalized to the maximal possible similarity score. Numbers, colours and shapes all indicate normalized similarity scores.

Figure 5 - Sparse principal component analysis with 6 components and 100 genes per component. A-C show individual components 1-6 with ellipses indicating 95\% confidence interval. D shows normalized expression values of genes present in the first 5 components with hierarchical clustering for both genes and datasets.

Figure 6-GSEA results of KEGG pathways significantly enriched in three or more datasets (see supplemental figure 5.1 for full list). Disease specific pathways are removed from the list.

Figure 7-Log ratios of genes in the two most upregulated (Proteasome [A] and RNA Transport [B]) and downregulated (Thermogenesis [C] and Oxidative Phosphorylation [D]) pathways. Hierarchical clustering is used for both experiments and genes.

Figure 8 - Correlation matrix of normalized enrichment scores of all KEGG pathways. Only significant correlations are shown (p<0.05). Experiments are ordered based on hierarchical clustering. Numbers, colours and shapes all indicate correlation coefficients.

Supplemental figure 1 - Full list GSEA results of KEGG pathways significantly expressed in more than one of the datasets. Some disease specific pathways are removed from the list.

Supplemental figure 2 - Heatmap of sPCA (6 components with each 25 genes) results of all samples, normalized per experiment. 


\section{References}

1. Fearon K, Arends J, Baracos V. Understanding the mechanisms and treatment options in cancer cachexia. Nat Rev Clin Oncol. Nature Publishing Group, a division of Macmillan Publishers Limited. All Rights Reserved.; 2013;10:90-9.

2. Witkamp RF, van Norren K. Let thy food be thy medicine.... when possible. Eur J Pharmacol. Elsevier B.V.; 2018;836:102-14.

3. Bonetto A, Aydogdu T, Kunzevitzky N, Guttridge DC, Khuri S, Koniaris LG, et al. STAT3 activation in skeletal muscle links muscle wasting and the acute phase response in cancer cachexia. PLoS One. 2011;6.

4. Cornwell EW, Mirbod A, Wu C-L, Kandarian SC, Jackman RW. C26 Cancer-Induced Muscle Wasting Is IKK $\beta$-Dependent and NF-kappaB-Independent. Gallouzi IE, editor. PLoS One. Public Library of Science; 2014;9:e87776.

5. Gilabert M, Calvo E, Airoldi A, Hamidi T, Moutardier V, Turrini O, et al. Pancreatic cancer-induced cachexia is Jak2dependent in mice. J Cell Physiol. 2014;229:1437-43.

6. Judge SM, Wu C-L, Beharry AW, Roberts BM, Ferreira LF, Kandarian SC, et al. Genome-wide identification of FoxOdependent gene networks in skeletal muscle during C26 cancer cachexia. BMC Cancer. 2014;14:997.

7. Shum AMYAAMY, Fung DCYY, Corley SM, McGill MC, Bentley NL, Tan TTC, et al. Cardiac and Skeletal Muscles Show Molecularly Distinct Responses to Cancer Cachexia. Physiol Genomics. American Physiological Society; 2015;47:physiolgenomics.00128.2014.

8. Fukawa T, Yan-Jiang BC, Min-Wen JC, Jun-Hao ET, Huang D, Qian C-N, et al. Excessive fatty acid oxidation induces muscle atrophy in cancer cachexia. Nat Med. Nature Publishing Group; 2016;22:666-71.

9. Goncalves MD, Hwang S-K, Pauli C, Murphy CJ, Cheng Z, Hopkins BD, et al. Fenofibrate prevents skeletal muscle loss in mice with lung cancer. Proc Natl Acad Sci. 2018;115:E743 LP-E752.

10. Blackwell TA, Cervenka I, Khatri B, Brown JL, Rosa-Caldwell ME, Lee DE, et al. A Transcriptomic Analysis of the Development of Skeletal Muscle Atrophy in Cancer-Cachexia in Tumor-Bearing Mice. Physiol Genomics. American Physiological Society; 2018;

11. Widner DB, Files DC, Weaver KE, Shiozawa Y. Preclinical and clinical studies on cancer-associated cachexia. Front Biol (Beijing). 2018;13:11-8.

12. Mueller TC, Bachmann J, Prokopchuk O, Friess H, Martignoni ME. Molecular pathways leading to loss of skeletal muscle mass in cancer cachexia - can findings from animal models be translated to humans? BMC Cancer. BioMed Central; 2015;16:75.

13. Irizarry RA, Hobbs B, Collin F, Beazer-barclay YD, Antonellis KJ, Speed TP. Exploration, Normalization , and Summaries of High Density Oligonucleotide Array Probe Level Data. 2018;249-64.

14. Dai M, Wang P, Boyd AD, Kostov G, Athey B, Jones EG, et al. Evolving gene/transcript definitions significantly alter the interpretation of GeneChip data. Nucleic Acids Res. 2005/11/15. 2005;33:e175.

15. Shi W, Oshlack A, Smyth GK. Optimizing the noise versus bias trade-off for Illumina whole genome expression BeadChips. Nucleic Acids Res. 2010;38.

16. Barbosa-Morais NL, Dunning MJ, Samarajiwa SA, Darot JFJ, Ritchie ME, Lynch AG, et al. A re-annotation pipeline for Illumina BeadArrays: Improving the interpretation of gene expression data. Nucleic Acids Res. 2009;38.

17. Chen Y, Lun ATL, Smyth GK. From reads to genes to pathways: differential expression analysis of RNA-Seq experiments using Rsubread and the edgeR quasi-likelihood pipeline. F1000Research. 2016;5:1438.

18. Robinson MD, McCarthy DJ, Smyth GK. edgeR: A Bioconductor package for differential expression analysis of digital gene expression data. Bioinformatics. 2009;26:139-40.

19. Robinson MD, Oshlack A. A scaling normalization method for differential expression analysis of RNA-seq data. Genome Biol. 2010;11:R25.

20. Law CW, Chen Y, Shi W, Smyth GK. voom: precision weights unlock linear model analysis tools for RNA-seq read counts. Genome Biol. 2014;15:R29.

21. Ritchie ME, Phipson B, Wu D, Hu Y, Law CW, Shi W, et al. Limma powers differential expression analyses for RNAsequencing and microarray studies. Nucleic Acids Res. 2015;43:e47.

22. Sartor MA, Tomlinson CR, Wesselkamper SC, Sivaganesan S, Leikauf GD, Medvedovic M. Intensity-based hierarchical Bayes method improves testing for differentially expressed genes in microarray experiments. BMC Bioinformatics. 2006;7:1-17.

23. Wilkinson L. ggplot2: Elegant Graphics for Data Analysis by WICKHAM, H. Biometrics. 2011;67:678-9.

24. Wei T, Simko V. R package "corrplot": Visualization of a Correlation Matrix (Version 0.84). Available from https://github.com/taiyun/corrplot; 2017.

25. Lottaz C, Yang X, Scheid S, Spang R. OrderedList - A bioconductor package for detecting similarity in ordered gene lists. Bioinformatics. 2006;22:2315-6.

26. Shannon P, Markiel A, Ozier O, Baliga NS, Wang JT, Ramage D, et al. Cytoscape: a software environment for integrated models of biomolecular interaction networks. Genome Res. 2003;13:2498-504.

27. Witten DM, Tibshirani R, Hastie T. A penalized matrix decomposition, with applications to sparse principal components and canonical correlation analysis. Biostatistics. 2009;10:515-34.

28. Shen $\mathrm{H}$, Huang JZ. Sparse principal component analysis via regularized low rank matrix approximation. J Multivar 
Anal. 2008;99:1015-34.

29. Subramanian A, Tamayo P, Mootha VK, Mukherjee S, Ebert BL, Gillette MA, et al. Gene set enrichment analysis: A knowledge-based approach for interpreting genome-wide expression profiles. Proc Natl Acad Sci. 2005;102:15545-50. 30. Kanehisa M, Sato Y, Kawashima M, Furumichi M, Tanabe M. KEGG as a reference resource for gene and protein annotation. Nucleic Acids Res. 2016;44:D457-62.

31. Yu G, Wang L-G, Han Y, He Q-Y. clusterProfiler: an R Package for Comparing Biological Themes Among Gene Clusters. Omi A J Integr Biol. 2012;16:284-7.

32. van der Ende M, Grefte S, Plas R, Meijerink J, Witkamp R, Keijer J, et al. Mitochondrial dynamics in cancer-induced cachexia. Biochim Biophys Acta - Rev Cancer. Elsevier; 2018;1870:137-50.

33. Argilés JM, Busquets S, Stemmler B, López-Soriano FJ. Cancer cachexia: understanding the molecular basis. Nat Rev Cancer. Nature Publishing Group, a division of Macmillan Publishers Limited. All Rights Reserved.; 2014;14:754-62.

34. Tisdale MJ. Mechanisms of Cancer Cachexia. Physiol Rev. 2009;89:381-410.

35. Acharyya S, Guttridge DC. Cancer cachexia signaling pathways continue to emerge yet much still points to the proteasome. Clin Cancer Res. 2007;13:1356-61.

36. Khal J, Wyke SM, Russell ST, Hine A V., Tisdale MJ. Expression of the ubiquitin-proteasome pathway and muscle loss in experimental cancer cachexia. Br J Cancer. Cancer Research UK; 2005;93:774-80.

37. Williams A, Sun X, Fischer JE, Hasselgren P-O. The expression of genes in the ubiquitin-proteasome proteolytic pathway is increased in skeletal muscle from patients with cancer. Surgery. 1999;126:744-50.

38. Gallagher IJ, Stephens NA, MacDonald AJ, Skipworth RJE, Husi H, Greig CA, et al. Suppression of skeletal muscle turnover in cancer cachexia: evidence from the transcriptome in sequential human muscle biopsies. Clin Cancer Res. American Association for Cancer Research; 2012;18:2817-27.

39. Constantinou C, Fontes de Oliveira CC, Mintzopoulos D, Busquets S, He J, Kesarwani M, et al. Nuclear magnetic resonance in conjunction with functional genomics suggests mitochondrial dysfunction in a murine model of cancer cachexia. Int J Mol Med. Spandidos Publications; 2011;27:15-24.

40. Östlund G, Sonnhammer ELL. Avoiding pitfalls in gene (co)expression meta-analysis. Genomics. Elsevier Inc.; 2014;103:21-30.

41. Jiang R, Zhao C, Gao B, Xu J, Song W, Shi P. Mixomics analysis of breast cancer: Long non-coding RNA linc01561 acts as ceRNA involved in the progression of breast cancer. Int J Biochem Cell Biol. Elsevier; 2018;102:1-9.

42. Rau A, Flister M, Rui H, Auer PL. Exploring drivers of gene expression in the Cancer Genome Atlas. Bioinformatics. 2018;35:62-8.

43. Warnat $P$, Eils R, Brors B. Cross-platform analysis of cancer microarray data improves gene expression based classification of phenotypes. BMC Bioinformatics. 2005;6:1-15.

44. Waldron L, Riester M. Meta-Analysis in Gene Expression Studies. Appl Environ Microbiol. 2016. p. 161-76.

45. Rappoport N, Shamir R. Multi-omic and multi-view clustering algorithms: review and cancer benchmark. Nucleic Acids Res. Oxford University Press; 2018;46:10546-62.

46. von Haehling S, Morley JE, Coats AJSS, Anker SD. Ethical guidelines for publishing in the journal of cachexia, sarcopenia and muscle: Update 2015. J Cachexia Sarcopenia Muscle. 2017;6:1081-3. 
Figures

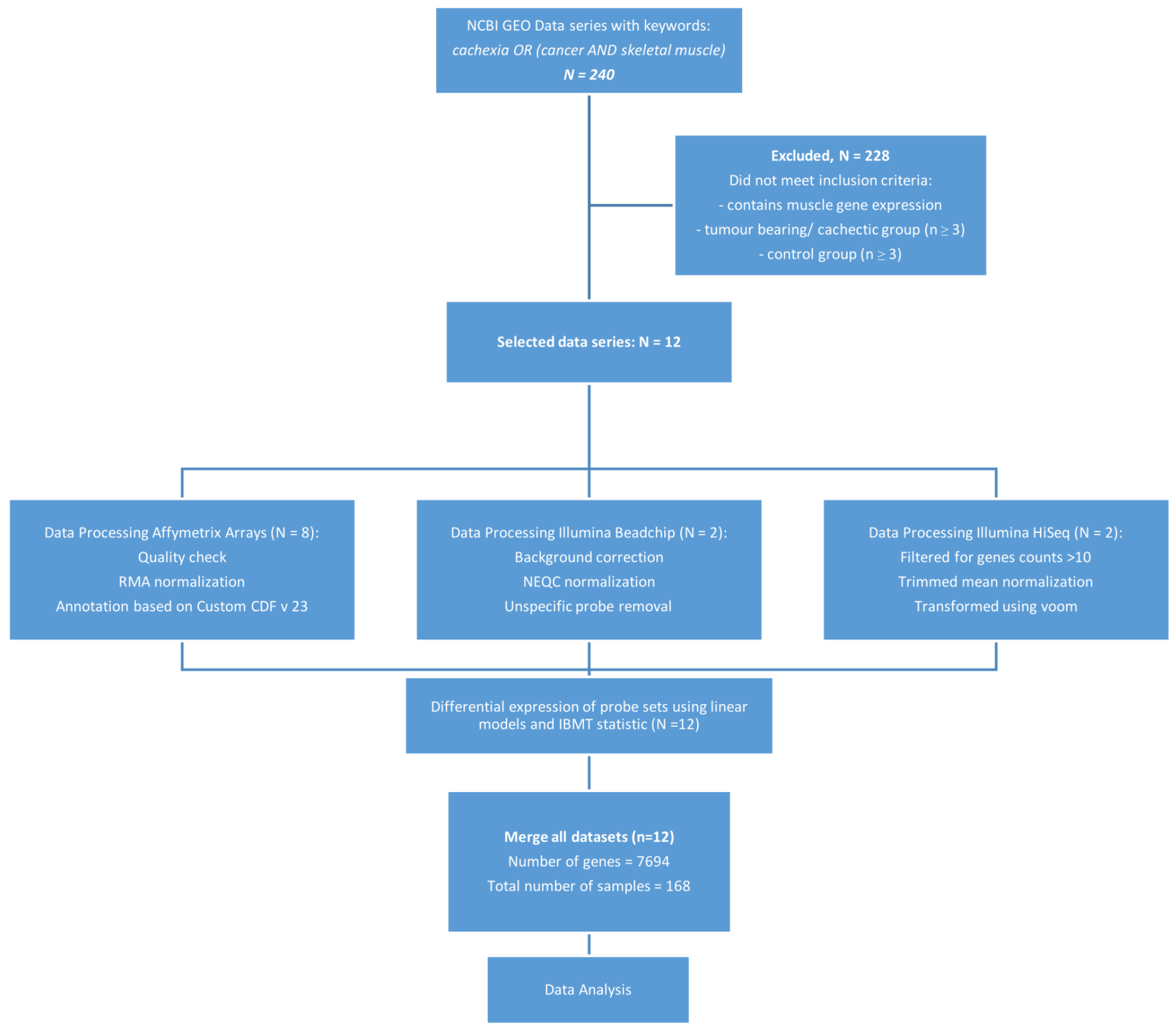

\section{Figure 1}

Flowchart of data series inclusion and data processing. 
Number of significantly changed genes per experiment

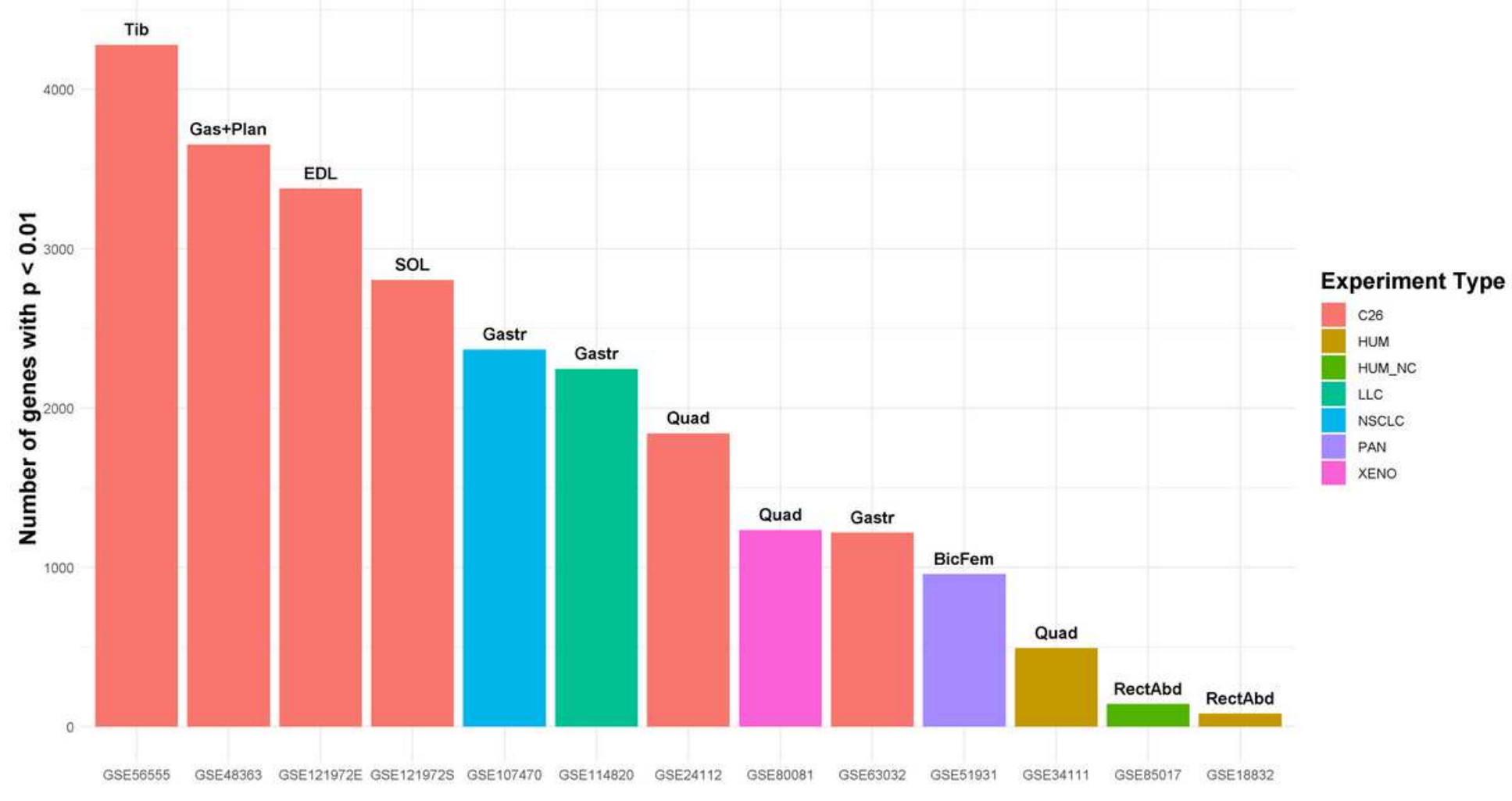

Figure 2

Number of significantly changed genes $(p<0.01)$ per experiment based on the complete dataset containing gene expression of 7694 genes present in all datasets. 


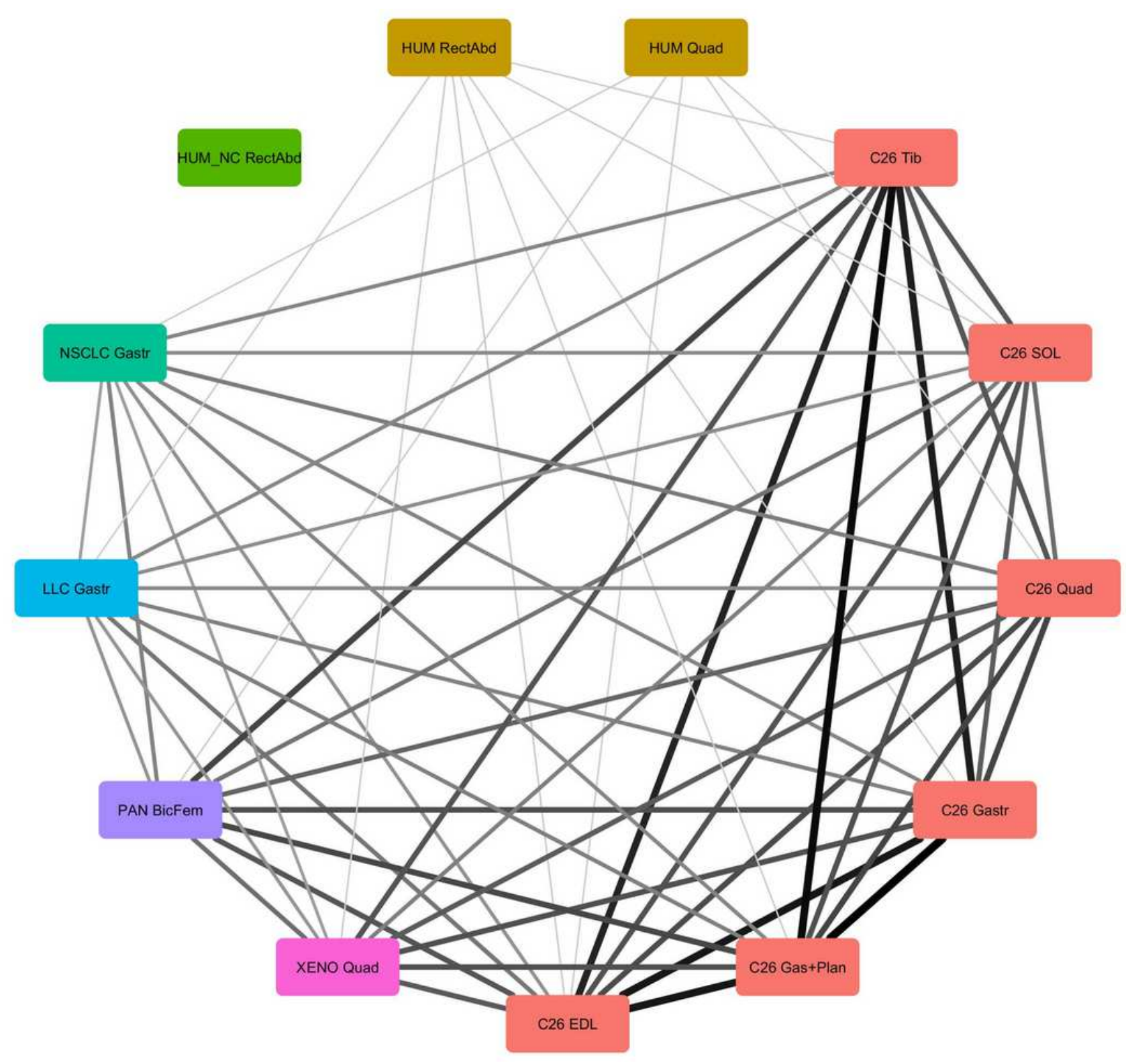

Figure 3

Network plot of similarity scores. Line width represent strength of similarity. Only similarities with $p<0.05$ are shown. 


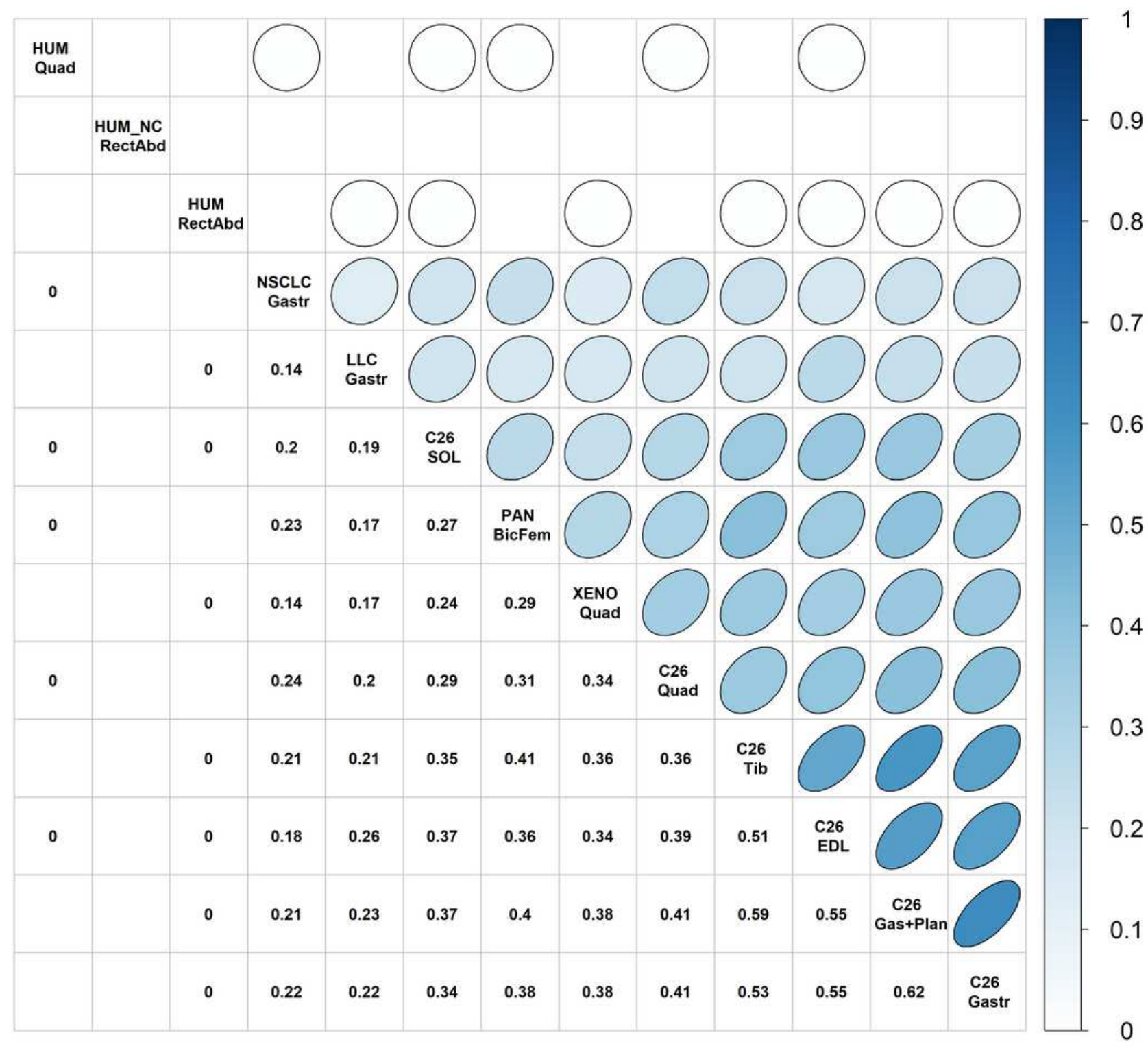

\section{Figure 4}

Similarity matrix showing normalized similarity with $p<0.05$. All similarity scores were normalized to the maximal possible similarity score. Numbers, colours and shapes all indicate normalized similarity scores. 

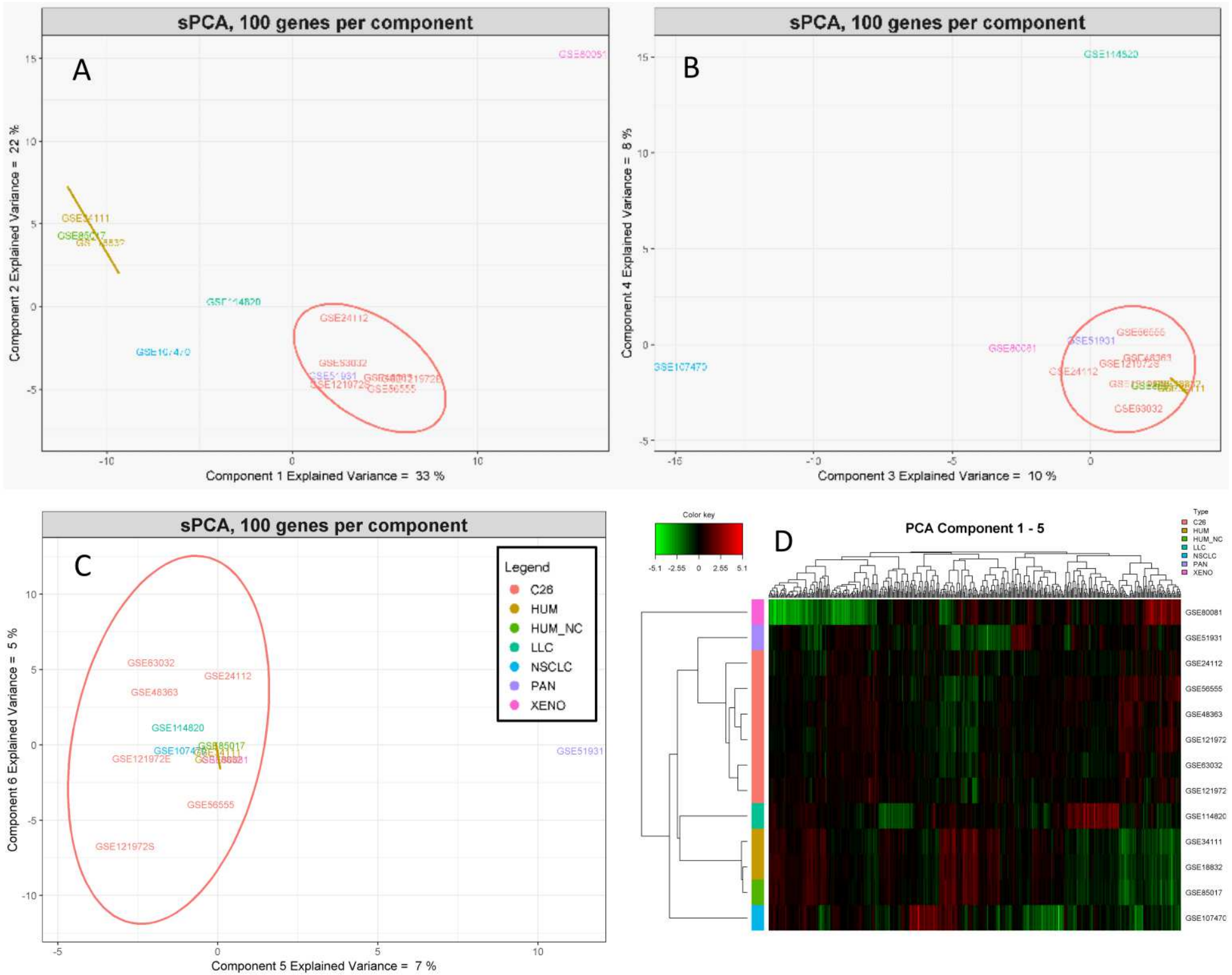

\section{Figure 5}

Sparse principal component analysis with 6 components and 100 genes per component. A-C show individual components 1-6 with ellipses indicating 95\% confidence interval. D shows normalized expression values of genes present in the first 5 components with hierarchical clustering for both genes and datasets. 


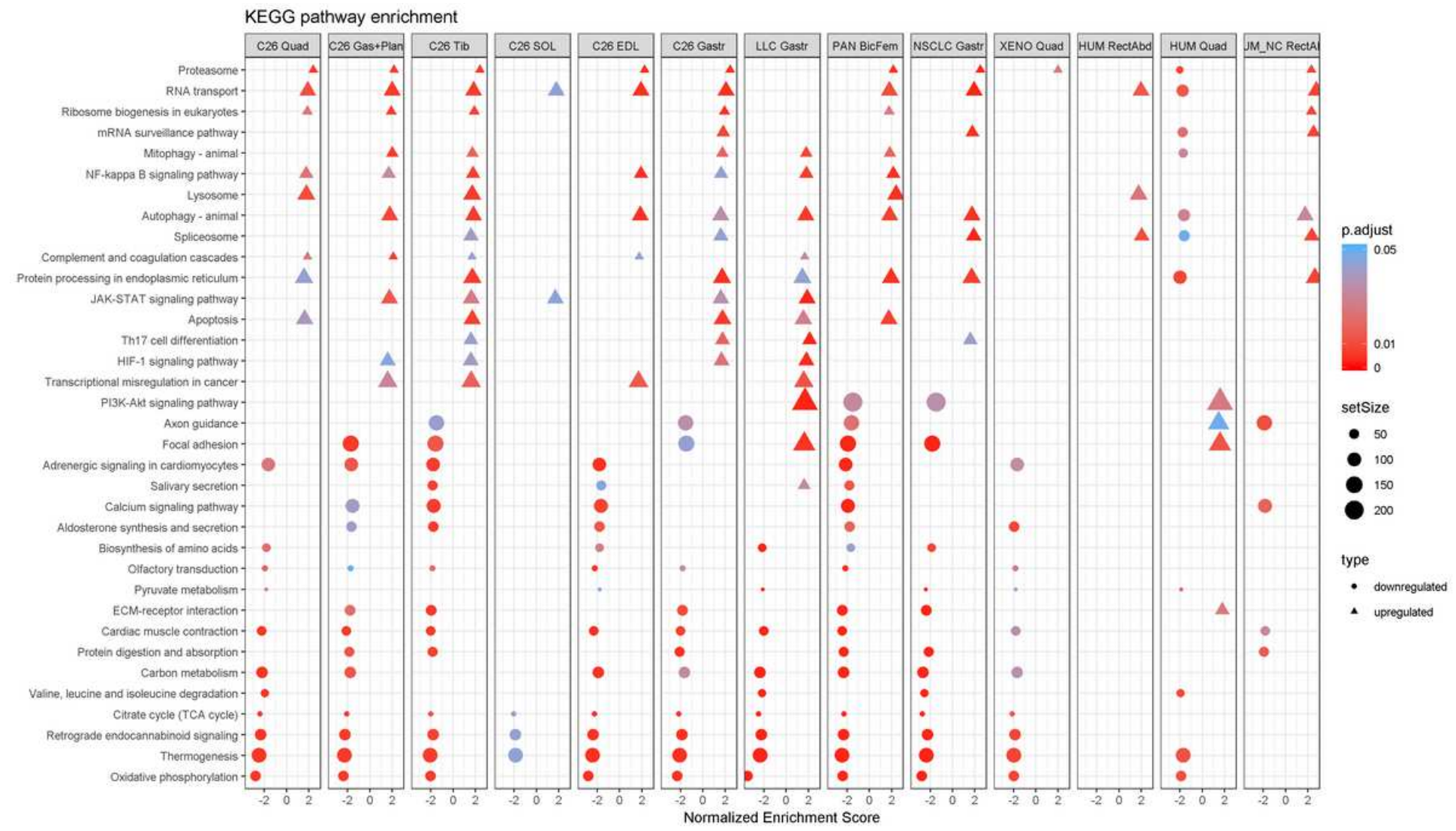

Figure 6

GSEA results of KEGG pathways significantly enriched in three or more datasets (see supplemental figure 5.1 for full list). Disease specific pathways are removed from the list. 

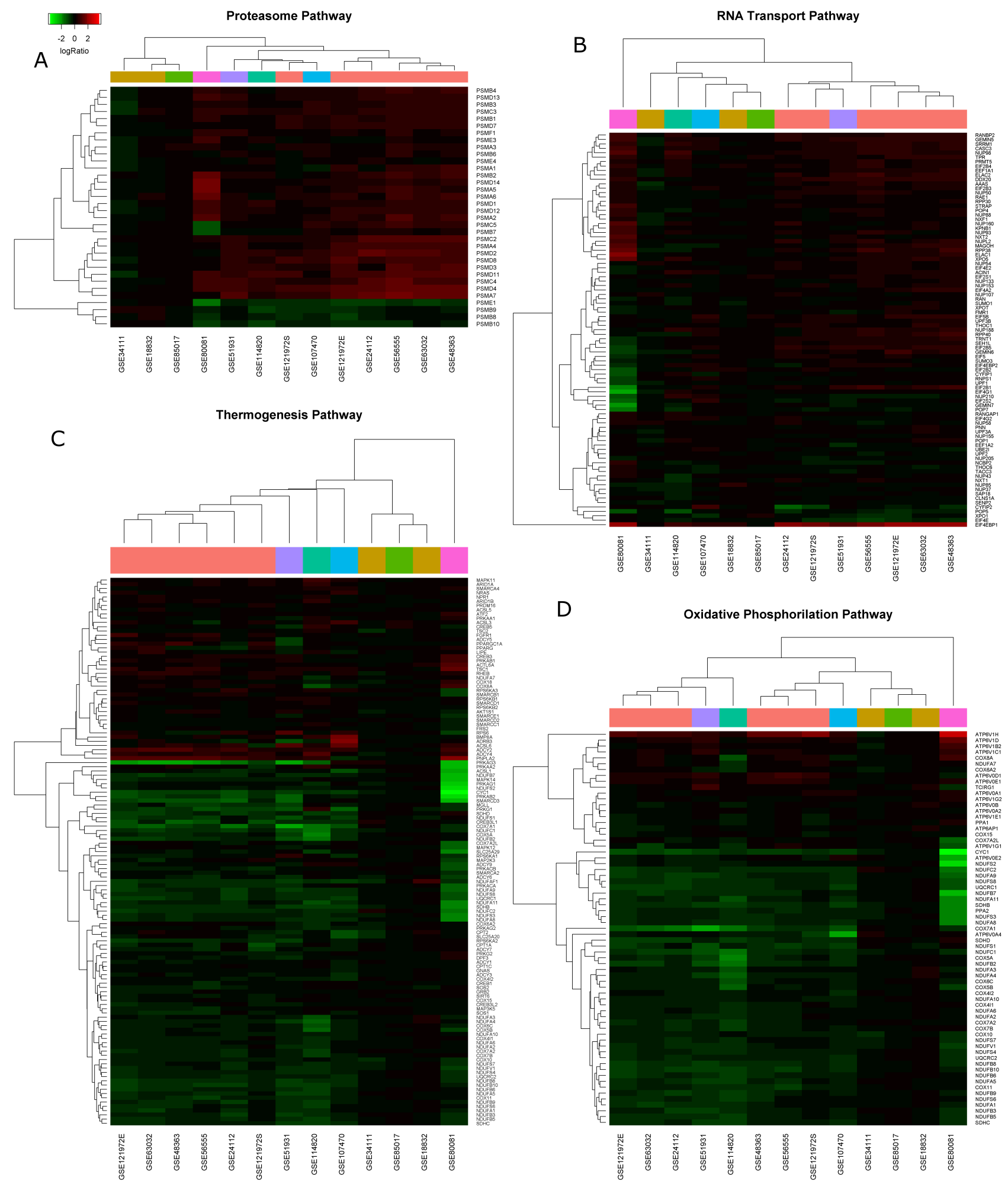

\section{Figure 7}

Log ratios of genes in the two most upregulated (Proteasome [A] and RNA Transport [B]) and downregulated (Thermogenesis [C] and Oxidative Phosphorylation [D]) pathways. Hierarchical clustering is used for both experiments and genes. 


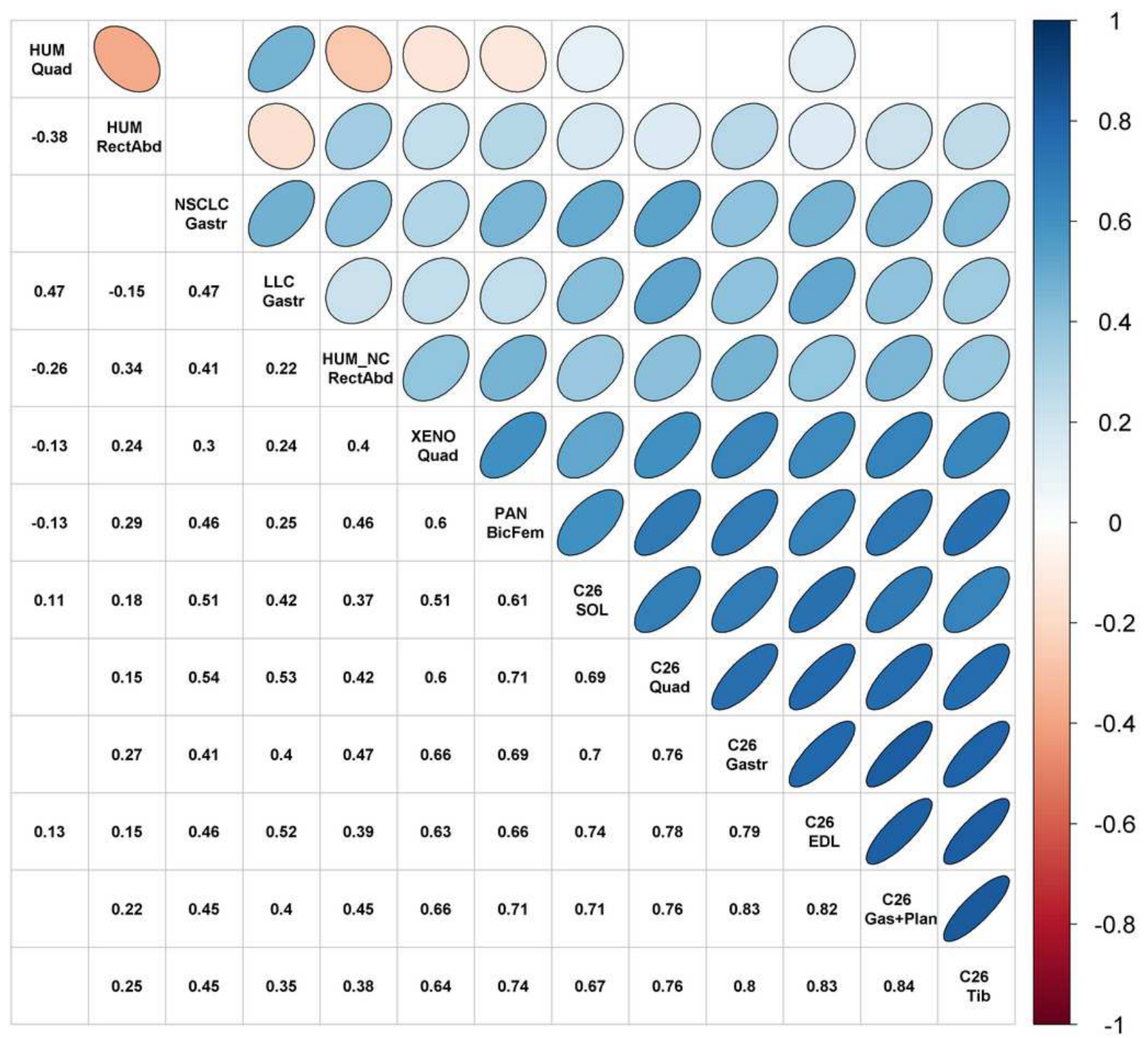

\section{Figure 8}

Correlation matrix of normalized enrichment scores of all KEGG pathways. Only significant correlations are shown $(p<0.05)$. Experiments are ordered based on hierarchical clustering. Numbers, colours and shapes all indicate correlation coefficients.

\section{Supplementary Files}


This is a list of supplementary files associated with this preprint. Click to download.

- HMDHumanPhenotype.txt

- EXPERIMENTOVERVIEW.csV

- 1.Installallpackages.R

- 2.AnalysisGSE121972.R

- 2.AnalysisGSE114820.R

- 2.AnalysisGSE107470.R

- 2.AnalysisGSE85017.R

- 2.AnalysisGSE63032.R

- 2.AnalysisGSE56555.R

- 2.AnalysisGSE51931.R

- 2.AnalysisGSE48363.R

- 2.AnalysisGSE34111.R

- 2.AnalysisGSE18832.R

- 2.AnalysisGSE24112GeneMean.R

- 2.AnalysisGSE80081GeneMean.R

- 3.PreprocessingArrayComparison.R

- 4.Analysisfinalfigures.R

- FigSup1.tiff

- FigSup2.tiff 